preprint SHEP-10-37

June 20, 2018

\title{
Low mass Higgs signals at the LHC in the Next-to-Minimal Supersymmetric Standard Model
}

\author{
M. M. Almarashi and S. Moretti \\ School of Physics and Astronomy, \\ University of Southampton, Southampton, SO17 1BJ, UK
}

\begin{abstract}
In the NMSSM, because of introducing a complex singlet superfield, the lightest CP-odd Higgs boson, $a_{1}$, can be a singlet-like state with a tiny doublet component in large regions of parameter space. In this paper, we examine the discovery potential of $a_{1}$ produced in association with a bottom-antibottom pair at the LHC through $\tau^{+} \tau^{-}$ and $\gamma \gamma$ decay modes. It is shown that an $a_{1}$ with mass $\leq M_{Z}$ can be extracted from the SM backgrounds by using the $\tau^{+} \tau^{-}$decay channel, a possibility precluded to the MSSM. In contrast, the $\gamma \gamma$ decay mode is overwhelmed by backgrounds despite the fact that the branching ratio of this mode can reach unity when $a_{1}$ is a pure singlet.
\end{abstract}

\section{Introduction}

In the Next-to-Minimal Supersymmetric Standard Model (NMSSM) [1], the soft Supersymmetry (SUSY)-breaking Higgs sector is described by the Lagrangian contribution

$$
V_{\mathrm{NMSSM}}=m_{H_{u}}^{2}\left|H_{u}\right|^{2}+m_{H_{d}}^{2}\left|H_{d}\right|^{2}+m_{S}^{2}|S|^{2}+\left(\lambda A_{\lambda} S H_{u} H_{d}+\frac{1}{3} \kappa A_{\kappa} S^{3}+\text { h.c. }\right),
$$

where $H_{u}$ and $H_{d}$ are the Higgs doublet fields, $S$ the singlet one, $\lambda$ and $\kappa$ Yukawa couplings while $A_{\lambda}$ and $A_{\kappa}$ are dimensionful parameters of order $M_{\mathrm{SUSY}}$, the typical SUSY mass scale.

As a result of the introduction of an extra complex singlet scalar field, which only couples to the two MSSM-type Higgs doublets, the Higgs sector of the NMSSM comprises of a total of seven mass eigenstates: a charged pair $h^{ \pm}$, three CP-even Higgses $h_{1,2,3}\left(m_{h_{1}}<m_{h_{2}}<m_{h_{3}}\right)$ 
and two CP-odd Higgses $a_{1,2}\left(m_{a_{1}}<m_{a_{2}}\right)$. Consequently, Higgs phenomenology in the NMSSM may be plausibly different from that of the MSSM and extremely rich of new signals.

For a start, the sum of the squares of the two lightest scalar Higgs boson masses is given by $[2]$ :

$$
m_{h_{1}}^{2}+m_{h_{2}}^{2} \approx M_{Z}^{2}+\frac{1}{2} \kappa<S>\left(4 \kappa<S>+\sqrt{2} A_{\kappa}\right)
$$

with $m_{h_{1}}^{2} \leq m_{h_{2}}^{2}$. The last expression can be translated into a modified upper bound of the $h_{1}$ mass as [2]

$$
m_{h_{1}}^{2} \lesssim \min \left\{M_{Z}^{2}, \frac{1}{2} \kappa<S>\left(4 \kappa<S>+\sqrt{2} A_{\kappa}\right)\right\}
$$

so that the upper bound on the NMSSM lightest Higgs boson mass is higher than the corresponding bound in the MSSM. Furthermore, as the higher order corrections are similar to those in the MSSM, also in higher orders the upper bound on the lightest Higgs boson mass remains different in the NMSSM with respect to the MSSM, reaching $140 \mathrm{GeV}$ or so, for maximal stop mixing and $\tan \beta=2$ [3, 4] (a configuration indeed excluded in the MSSM by LEP data). More in general, the 'little fine tuning problem', resulting in LEP failing to detect a light CP-even Higgs boson, predicted over most of the MSSM parameter space, is much attenuated in the NMSSM, either because a SM-like Higgs can decay dominantly into $a_{1} a_{1}[5]$ or because the mixing among more numerous $\mathrm{CP}$-even or CP-odd Higgs fields enables light mass states being produced at LEP or Tevatron yet, they can remain undetected because of their reduced couplings to $Z$ bosons [4]. (See Refs. [6] and [7] for benchmark points with very light CP-odd Higgs bosons in both the unconstrained and constrained NMSSM parameter space, respectively).

As for present accelerator machines, chiefly the Tevatron at FNAL and the Large Hadron Collider (LHC) at CERN, quite some work has been dedicated to probing the NMSSM Higgs sector over recent years. Primarily, there have been attempts to extend the so-called 'No-lose theorem' of the MSSM - stating that at least one MSSM Higgs boson should be observed via the usual SM-like production and decay channels at the LHC throughout the entire MSSM parameter space [8] - to the case of the NMSSM [6, 9, 10, 12, 13]. From this perspective, it was realised that at least one NMSSM Higgs boson should remain observable at the LHC over the NMSSM parameter space that does not allow any Higgs-to-Higgs decay. However, when the only light non-singlet (and, therefore, potentially visible) CP-even Higgs boson, $h_{1}$ or $h_{2}$, decays mainly to two very light CP-odd Higgs bosons, $h \rightarrow a_{1} a_{1}$, one may not have a Higgs signal of statistical significance at the LHC [14]. In fact, further violations to the theorem may well occur if one enables Higgs-to-SUSY decays (e.g., into neutralino pairs, yielding invisible Higgs signals).

While there is no definitive evidence on whether a 'No-lose theorem' can be proved for the NMSSM, we are here also concerned with an orthogonal approach. We asked ourselves if a, so to say, 'More-to-gain theorem' can be formulated in the NMSSM. That is, whether 
there exist regions of the NMSSM parameter space where more and/or different Higgs states of the NMSSM are visible at the LHC than those available within the MSSM. In our attempt to overview such a possibility, we consider here the case of the di-photon and $\tau^{+} \tau^{-}$decay channels of a light neutral Higgs boson. The first mode can successfully be probed in the MSSM, but limitedly to the case of the lightest CP-even Higgs boson and for masses of order $100-130 \mathrm{GeV}$ or so. We will show that in the NMSSM there exist regions of its parameter space where one can potentially have a sizable di-photon signal from a Higgs state of different nature (CP-odd instead of CP-even) and with much smaller mass (down to $10 \mathrm{GeV}$ or so), owing in large part to an increased $\operatorname{BR}\left(a_{1} \rightarrow \gamma \gamma\right)$. Such a possibility emerges in the NMSSM due to the fact that such a CP-odd Higgs state has a predominant singlet component and a very weak doublet one. As a consequence, all partial decay widths are heavily suppressed as they employ only the doublet component, except one: the $\gamma \gamma$ partial decay width. This comes from the fact that the $a_{1} \tilde{\chi}^{+} \tilde{\chi}^{-}$coupling is not suppressed, as it is generated through the $\lambda H_{1} H_{2} S$ Lagrangian term and therefore implies no small mixing. Although the direct decay $a_{1} \rightarrow \tilde{\chi}^{+} \tilde{\chi}^{-}$is forbidden, the aforementioned coupling participates in the $a_{1} \gamma \gamma$ effective coupling. The $\tau^{+} \tau^{-}$channel is used in the MSSM as a search channel of rather heavy and degenerate $\mathrm{CP}$-even and $\mathrm{CP}$-odd states and its exploitation has not been proved at very low masses, say, below $M_{Z}$. We will show here that the $\tau$-pair decay can be very relevant for such a mass interval, in the case of an $a_{1}$ state of the NMSSM. The extraction of either channel for low Higgs masses would then unmistakably point to the existence of non-minimal SUSY Higgs sector. We build on the results presented in [10] (see also [11]), where several such signals (for rather heavy Higgs states though) were established in some (complementary) regions of the NMSSM parameter space.

The plan of this paper is as follows. In Sec. 2 we describe the parameter space scans performed in order to isolate the combinations of NMSSM inputs that can give rise to the described phenomenology. In Sec. 3 we discuss typical event rates, highlighting the relevance of Higgs production in association with bottom quark pairs as most favourable production mode. In Sec. 4 we describe the signal-to-background analysis performed and introduce some benchmark points where an $a_{1}$ signal can be extracted (albeit limitedly to the $\tau^{+} \tau^{-}$decay mode). Finally, in Sec. 5 we summarise and conclude.

\section{Parameter Space Scan}

For a general study of the NMSSM Higgs sector, we used here the up-to-date publicly available fortran package NMSSMTools developed in Refs. [15, 16 1]. This package computes the masses, couplings and decay widths of all the Higgs bosons of the NMSSM in terms of its parameters at the Electro-Weak (EW) scale. The NMSSMTools also takes into account theoretical as well as experimental constraints from negative Higgs searches at LEP [17] and the Tevatron 2 , along with the unconventional channels relevant for the NMSSM.

For our purpose, instead of postulating unification, and without taking into account the SUSY breaking mechanism, we fixed the soft SUSY breaking terms to very high values, so

\footnotetext{
${ }^{1}$ We have used NMSSMTools_2.3.1.

${ }^{2}$ Speculations of an excess at LEP which could be attributed to NMSSM Higgs bosons are found in 18 .
} 
that they have little or no contribution to the outputs of the parameter scans. Consequently, we are left with the aforementioned six free parameters. Our parameter space is in particular defined through the Yukawa couplings $\lambda$ and $\kappa$, the soft trilinear terms $A_{\lambda}$ and $A_{\kappa}$, plus $\tan \beta$ (the ratio of the Vacuum Expectation Values (VEVs) of the two Higgs doublets) and $\mu_{\mathrm{eff}}=\lambda\langle S\rangle$ (where $\langle S\rangle$ is the VEV of the Higgs singlet). In our numerical analyses we have taken $m_{b}^{\text {pole }}=5 \mathrm{GeV}$ and $m_{t}^{\text {pole }}=171.4 \mathrm{GeV}$ for the bottom- and top-quark mass, respectively.

We have used the NMHDECAY code to scan over the NMSSM parameter space defined through the six parameters already discussed taken in the following intervals:

$$
\begin{gathered}
\lambda: 0.0001-0.7, \quad \kappa: 0-0.65, \quad \tan \beta: 1.6-54 \\
\mu: 100-1000 \mathrm{GeV}, \quad A_{\lambda}:-1000-+1000 \mathrm{GeV}, \quad A_{\kappa}:-10-0 .
\end{gathered}
$$

(Notice that our aim is exploring the parameter space which has very low $m_{a_{1}}$ and one way to do that is by choosing $A_{\kappa}$ small, in which case its negative value is preferred [2]).

Remaining soft terms which are fixed in the scan include:

- $m_{Q_{3}}=m_{U_{3}}=m_{D_{3}}=m_{L_{3}}=m_{E_{3}}=1 \mathrm{TeV}$,

- $A_{U_{3}}=A_{D_{3}}=A_{E_{3}}=1.2 \mathrm{TeV}$,

- $m_{Q}=m_{U}=m_{D}=m_{L}=m_{E}=1 \mathrm{TeV}$,

- $M_{1}=M_{2}=M_{3}=1.5 \mathrm{TeV}$.

As intimated, we have fixed soft term parameters at the TeV scale to minimise their contributions to parameter space outputs but changing values of some of those parameters such as $A_{U_{3}}$ could decrease or increase the number of successful points emerging from the NMSSMTools scans but without a significant impact on the $m_{a_{1}}$ distribution. Also, notice that the sfermion mass parameters and the $S U(2)$ gaugino mass parameter, $M_{2}$, play crucial roles in constraining $\tan \beta$. Lowering values of those parameters allow less values of $\tan \beta$ to pass experimental and theoretical constraints, however, this is a less interesting region of the NMSSM parameter space for our analysis, as our Higgs production mode is only relevant at large values of this parameter. The effect of heavy gaugino mass parameters on the output of parameter space, in particular $m_{a 1}$, would be small except for $M_{2}$ through its effect on $\tan \beta$.

In line with the assumptions made in [6, 9], the allowed decay modes for neutral NMSSM Higgs bosons are 3 :

$$
\begin{array}{cll}
h, a \rightarrow g g, \quad h, a \rightarrow \mu^{+} \mu^{-}, & h, a \rightarrow \tau^{+} \tau^{-}, & h, a \rightarrow b \bar{b}, \quad h, a \rightarrow t \bar{t}, \\
h, a \rightarrow s \bar{s}, \quad h, a \rightarrow c \bar{c}, & h \rightarrow W^{+} W^{-}, \quad h \rightarrow Z Z \\
h, a \rightarrow \gamma \gamma, \quad h, a \rightarrow Z \gamma, \quad h, a \rightarrow \text { Higgses, } & h, a \rightarrow \text { sparticles. }
\end{array}
$$

(Notice that for the CP-odd Higgses, the decay into vector boson pairs is not allowed due to CP-conservation). Here, 'Higgses' refers to any final state involving all possible combination of two Higgs bosons (neutral and/or charged) or of one Higgs boson and a gauge vector.

\footnotetext{
${ }^{3}$ Here, we use the label $h(a)$ to signify any of the neutral CP-even(odd) Higgs bosons of the NMSSM.
} 
We have performed our scan over 10 million of randomly selected points in the specified parameter space. The output, as stated earlier, contains masses, Branching Ratios (BRs) and couplings of the NMSSM Higgses, for all the points which are not forbidden by the various experimental and theoretical constraints. The points which violate the constraints are eliminated.

\section{Inclusive Event Rates}

The surviving data points are then used to determine the cross sections for NMSSM Higgs hadro-production by using CalcHEP [19], wherein some new modules have been implemented for this purpose [21. As the SUSY mass scale has been arbitrarily set well above the EW one (see above), the NMSSM Higgs production modes exploitable in simulations at the LHC are those involving couplings to heavy ordinary matter only, i.e., (hereafter, $V=W^{ \pm}, Z$ and $Q=b, t$ ) for neutral Higgs production (where the last two channels are only allowed for CP-even Higgs production):

$$
\begin{gathered}
g g \rightarrow \text { Higgs (gluon - fusion, via heavy - quark loops), } \\
q \bar{q}, g g \rightarrow Q \bar{Q} \text { Higgs (heavy - quark associated production), } \\
q q \rightarrow q q V^{*} V^{*} \rightarrow q q \text { Higgs (Vector Boson Fusion (VBF)), } \\
q \bar{q} \rightarrow V \text { Higgs (Higgs - strahlung). }
\end{gathered}
$$

(These are the so-called 'direct' Higgs production modes). Here, 'Higgs' refers to any possible neutral Higgs boson. We can however anticipate that, for the purpose of extracting an $a_{1} \rightarrow \gamma \gamma$ or $a_{1} \rightarrow \tau^{+} \tau^{-}$resonance, only the second channel turns out to be useful and limitedly to the case $Q=b$. This is because the associated $b \bar{b}$ pairs can be (vertex) tagged, thus offering a useful handle for background rejection. The case $Q=t$ in fact gives too small cross section. The gluon fusion channel is instead burdened by huge Standard Model (SM) backgrounds $(q \bar{q}, g g \rightarrow \gamma \gamma$ as well as jets mis-identified as photons in the $\gamma \gamma$ mode and Drell-Yan and di-jet production in the $\tau^{+} \tau^{-}$channel). Higgs-strahlung and VBF also suffer from large SM backgrounds, though their main drawback are rather smaller production rates in comparison (as they are EW processes).

In the NMSSM, $a_{1}$ state is a composition of the usual doublet component of the CP-odd MSSM Higgs boson, $a_{\mathrm{MSSM}}$, and the new singlet component, $a_{\mathrm{S}}$, coming from the singlet superfield of the NMSSM. This can be written as [18]:

$$
a_{1}=a_{\mathrm{MSSM}} \cos \theta_{A}+a_{\mathrm{S}} \sin \theta_{A} .
$$

For very small values of $A_{k}$, the lightest CP-odd Higgs, $a_{1}$, is mostly singlet-like with a tiny doublet component, i.e. the mixing angle $\cos \theta_{A}$ is small, see top-pane of Fig. 1, which shows the relation between $m_{a_{1}}$ and $\cos \theta_{A}$. If $a_{1}$ is highly singlet, $\cos \theta \sim 0$, then $\operatorname{BR}\left(a_{1} \rightarrow \gamma \gamma\right)$

\footnotetext{
${ }^{4}$ We adopt herein CTEQ6L [20] as parton distribution functions, with scale $Q=\sqrt{\hat{s}}$, the centre-of-mass energy at parton level, for all processes computed.
} 
can reach unity as shown in the bottom-pane of this figure.

To good approximation, $m_{a_{1}}$ can be written in the NMSSM as [18]:

$$
m_{a_{1}}^{2}=-3 \frac{\kappa A_{\kappa} \mu_{\mathrm{eff}}}{\lambda} \sin ^{2} \theta_{A}+\frac{9 A_{\lambda} \mu_{\mathrm{eff}}}{2 \sin 2 \beta} \cos ^{2} \theta_{A} .
$$

The first term of the this expression is dominant especially at large $\tan \beta$. Also, it is clear that a combination of all tree level Higgs sector parameters jointly affects $m_{a_{1}}$ in general.

As an initial step towards the analysis of the data, we have computed $m_{a_{1}}$ against each of the six tree level Higgs sector parameters of the NMSSM. Fig. 2 presents the results of our scan, these series of plots illustrating the distribution of $m_{a_{1}}$ over the six parameters and as a function of $\operatorname{BR}\left(a_{1} \rightarrow \gamma \gamma\right)$ and $\operatorname{BR}\left(a_{1} \rightarrow \tau^{+} \tau^{-}\right)$. In the parameter space adopted for this analysis we have noticed that the large $\tan \beta$ and small $\mu_{\mathrm{eff}}$ (and, to some extent, also $\lambda$ ) region is the one most compatible with current theoretical and experimental constraints, though this conclusion should not be generalised to the entire parameter space. Herein, it is also obvious that $m_{a_{1}}$ increases by increasing $\kappa$ and $-A_{\kappa}$ whereas it decreases by increasing $A_{\lambda}$. Moreover, from a closer look at Fig. 2, it is clear that the $\operatorname{BR}\left(a_{1} \rightarrow \gamma \gamma\right)$ can be very large, indeed dominant, and $m_{a_{1}}$ values in the region 50 to $100 \mathrm{GeV}$ maximise that rate. Also, notice that $\operatorname{BR}\left(a_{1} \rightarrow \tau^{+} \tau^{-}\right)$reaches about $10 \%$ in most of the parameter space which has $m_{a_{1}} \geq 10 \mathrm{GeV}$, in which case the $a_{1}$ decay into $b \bar{b}$ is open and dominant. Yet also notice that there is a small region with very low $m_{a_{1}}$ values, $m_{a_{1}}<10 \mathrm{GeV}$, yielding a large $\operatorname{BR}\left(a_{1} \rightarrow \tau^{+} \tau^{-}\right)$, greater than $90 \%$, as shown in the bottom-right corner of the bottomright pane of this figure. The latter region occurs when $a_{1} \rightarrow b \bar{b}$ is closed, in which case $\operatorname{BR}\left(a_{1} \rightarrow \tau^{+} \tau^{-}\right)$is dominant compared to $c \bar{c}, \mu^{+} \mu^{-}$, et $[5$.

Fig. 3 shows the distribution of event rates, $\sigma\left(g g \rightarrow b \bar{b} a_{1}\right) \operatorname{BR}\left(a_{1} \rightarrow \gamma \gamma\right)$ and $\sigma(g g \rightarrow$ $\left.b \bar{b} a_{1}\right) \operatorname{BR}\left(a_{1} \rightarrow \tau^{+} \tau^{-}\right)$as functions of $m_{a_{1}}$, BR of the corresponding channel and $\tan \beta$. As expected, the inclusive cross section decreases with increasing $m_{a_{1}}$, see the top two panes of this figure. Although the $\operatorname{BR}\left(a_{1} \rightarrow \gamma \gamma\right)$ can be dominant over a sizable expanse of the NMSSM parameter space (middle-left pane), the latter does not correspond to the region that maximises the yield of $\sigma\left(g g \rightarrow b \bar{b} a_{1}\right) \mathrm{BR}\left(a_{1} \rightarrow \gamma \gamma\right)$, as the maximum of the latter occurs for BRs in the region of a few $10^{-5}$ to $10^{-4}$. Therefore, one cannot take full advantage of the phenomenon described in the introduction, with respect to the singlet nature of the $a_{1}$ state entering the $a_{1} \tilde{\chi}^{+} \tilde{\chi}^{-}$coupling, as the doublet component (necessary to enable a large $a_{1} b \bar{b}$ coupling at production level) plays a stronger role in comparison. The tension between the two trends is such that the cross section times BR rates are less than $100 \mathrm{fb}$. The outlook for the $\tau^{+} \tau^{-}$case is much brighter, as corresponding signal rates are at nb level for $\operatorname{BR}\left(a_{1} \rightarrow \tau^{+} \tau^{-}\right) \approx 0.1$ or even $10 \mathrm{nb}$ for $\operatorname{BR}\left(a_{1} \rightarrow \tau^{+} \tau^{-}\right) \approx 1$, see middle-right pane of Fig. 3. Also notice that such large rates naturally occur for any $m_{a_{1}}$ in the allowed interval (see top-right pane of this figure).

Incidentally, notice in the case of both decay channels that not only the density of NMSSM

\footnotetext{
${ }^{5}$ Notice that the mass region below the $b \bar{b}$ threshold is severely constrained, see, e.g., Ref. [22] (and references therein).
} 
parameter configurations is larger as $\tan \beta$ grow 6 but also the event rates are maximal at large values of this parameter (see bottom-left and bottom-right panes of Fig. 3), thereby confirming what we intimated at the beginning of this section about the relevance of the $q \bar{q}, g g \rightarrow b \bar{b} a_{1}$ production mode (whose cross section is essentially proportional to $\tan ^{2} \beta$ ).

In the NMSSM, there is a large area of parameter space where one Higgs state can decay into two, e.g., $h_{1} \rightarrow a_{1} a_{1}$ : see Fig. 4. As it is clear from the left-pane of this figure, the majority of points generated here have $m_{h 1}>110 \mathrm{GeV}$ and $m_{a_{1}}<55 \mathrm{GeV}$, thereby allowing the possibility of $h_{1} \rightarrow a_{1} a_{1}$ decays. Moreover, this decay can be dominant and can reach unity as shown in the right-pane of this figure. Despite this, such a decay may not give Higgs signals with sufficient statistical significance at the LHC (as discussed in previous literature and recalled here previously). Therefore, we are here well motivated to look further at the scope of direct production of $a_{1}$ state in single mode at the LHC, through $g g \rightarrow b \bar{b} a_{1}$, over overlapping regions of NMSSM parameter space, which we are going to do next.

\section{Signal-to-Background Analysis}

We perform here a partonic signal-to-background $(S / B)$ analysis, based on CalcHEP, in the two channels $\gamma \gamma$ and $\tau^{+} \tau^{-}$separately in the two forthcoming subsections. We assume $\sqrt{s}=14 \mathrm{TeV}$ throughout for the LHC energy and we will benchmark event rates on the basis of 300 inverse femtobarn of accumulated luminosity.

From the output of NMSSMTools, we have chosen some points which have large (yet not maximal) event rates as illustrative examples to test the possibility of detecting $a_{1}$ with different masses at the LHC. They are located in large regions of parameter space with high density. These illustrative points are as follows:

- For $m_{a_{1}}=9.8 \mathrm{GeV}$ :

$\lambda=0.22341, \kappa=0.41849, \tan \beta=53.82, \mu=228.94, A_{\lambda}=-415.57$ and $A_{\kappa}=-6.18$.

- For $m_{a_{1}}=20 \mathrm{GeV}$ :

$\lambda=0.07595, \kappa=0.11544, \tan \beta=51.51, \mu=377.44, A_{\lambda}=-579.64$ and $A_{\kappa}=-3.53$.

- For $m_{a_{1}}=31 \mathrm{GeV}$ :

$\lambda=0.10861, \kappa=0.46542, \tan \beta=48.06, \mu=222.99, A_{\lambda}=-952.6$ and $A_{\kappa}=-7.21$.

- For $m_{a_{1}}=46 \mathrm{GeV}$ :

$\lambda=0.14088, \kappa=0.25219, \tan \beta=50.56, \mu=317.08, A_{\lambda}=-569.61$ and $A_{\kappa}=-8.61$.

- For $m_{a_{1}}=60.5 \mathrm{GeV}$ :

$\lambda=0.17411, \kappa=0.47848, \tan \beta=52.39, \mu=169.83, A_{\lambda}=-455.85$ and $A_{\kappa}=-9.03$.

- For $m_{a_{1}}=81 \mathrm{GeV}$ :

$\lambda=0.10713, \kappa=0.13395, \tan \beta=44.72, \mu=331.43, A_{\lambda}=-418.13$ and $A_{\kappa}=-9.71$.

\footnotetext{
${ }^{6}$ Again, notice that for a more general choice of the range of $A_{\kappa}$ and values of the soft SUSY breaking parameters this would not necessarily hold.
} 


\subsection{The $\gamma \gamma$ channel}

Altogether, the fact that production cross section and BR decay are maximised each in different regions of the NMSSM parameter space makes it extremely difficult to obtain detectable event rates in this case. In fact, the signal yields for this channel are not only small in general, but also overwhelmed by the irreducible background. This is made explicit in Figs. 5-6, which show how the signal, despite yielding sizable peaks in the di-photon invariant mass $m_{\gamma \gamma}$ (for example, for all $m_{a_{1}}$ masses considered - between $\approx 10$ and $\approx 46 \mathrm{GeV}$ - one can obtain some $\mathcal{O}(10)$ events every $300 \mathrm{fb}^{-1}$, see top-left plot of each figure), is completely spoilt by the irreducible background, after using standard cut:7:

$$
\begin{gathered}
\Delta R(b, \bar{b}), \Delta R(b, \gamma), \Delta R(\bar{b}, \gamma), \Delta R(\gamma, \gamma)>0.4 \\
|\eta(b)|,|\eta(\bar{b})|,|\eta(\gamma)|<2.5 \\
P_{T}(b), P_{T}(\bar{b})>20 \mathrm{GeV}, P_{T}(\gamma)>2 \mathrm{GeV} .
\end{gathered}
$$

In fact, for triggering purposes, at least one of the two photon transverse momenta ought to be increased to some $10 \mathrm{GeV}$ or so, which does not help to improve $S / B$, as can be appreciated in the top-right and bottom plots in Figs. 5-6, which show the average transverse momentum of a photon as well as its minimum and maximum, respectively. For $a_{1}$ masses above $50 \mathrm{GeV}$ or so the signal rates are (in general) too poor to even pass the observability threshold of 1 event after $300 \mathrm{fb}^{-1}$.

In summary, despite the uniqueness of this signal, with the potential of clearly highlighting a possible singlet nature of the lightest CP-odd Higgs of the NMSSM, the latter is not extractable at the LHC.

\subsection{The $\tau^{+} \tau^{-}$channel}

The situation is instead much rosier for the $\tau^{+} \tau^{-}$channel. After implementing the following standard cut:8

$$
\begin{gathered}
\Delta R(b, \bar{b}), \Delta R\left(b, \tau^{+}\right), \Delta R\left(\bar{b}, \tau^{+}\right), \Delta R\left(b, \tau^{-}\right), \Delta R\left(\bar{b}, \tau^{-}\right), \Delta R\left(\tau^{+}, \tau^{-}\right)>0.4 \\
|\eta(b)|,|\eta(\bar{b})|,\left|\eta\left(\tau^{+}\right)\right|,\left|\eta\left(\tau^{-}\right)\right|<2.5 \\
P_{T}(b), P_{T}(\bar{b})>20 \mathrm{GeV}, P_{T}\left(\tau^{+}\right), P_{T}\left(\tau^{-}\right)>10 \mathrm{GeV}
\end{gathered}
$$

we obtain the invariant masses of the $\tau^{+} \tau^{-}$system depicted in Figs. 7-12 (see their lefthand sides), where the signal clearly appears over the irreducible background due to $q \bar{q}, g g \rightarrow$ $b \bar{b} \gamma, Z \rightarrow b \bar{b} \tau^{+} \tau^{-}$. Recalling that for low Higgs masses, say below $M_{Z}$, in the $b \bar{b} \tau^{+} \tau^{-}$channel, the dominant background is indeed the irreducible one, see, e.g., Ref. [23], at least in the double- and single-leptonic channels, it is clear that there exist substantial discovery potential of a very light CP-odd Higgs boson of the NMSSM at the LHC.

\footnotetext{
${ }^{7}$ Hereafter, $\eta$ refers to the pseudorapidity and $\Delta R$ to the cone distance expressed in differences of pseudorapidity and azimuthal angle $\phi$ : i.e., $\Delta R=\sqrt{(\Delta \eta)^{2}+(\Delta \phi)^{2}}$. Further, the notation $P_{T}$ refers to the transverse momentum.

${ }^{8}$ Here, for the sake of illustration, we take the $\tau$ 's to be on shell.
} 
For the sake of completeness, we have also shown in Figs. 7-12 the top-antitop reducible background, i.e., $q \bar{q}, g g \rightarrow t \bar{t} \rightarrow b \bar{b} W^{+} W^{-} \rightarrow b \bar{b} \tau^{+} \tau^{-} P_{T}^{\text {miss }}$ (with no cuts on $P_{T}^{\text {miss }}$ though, which could always be enforced for its suppression), making it clear that discovery of heavier $a_{1}$ states in $\tau^{+} \tau^{-}$pairs, even in the the double- and single-leptonic channels, becomes much more difficult, although not impossible (also owing to the long $Z$ tail in the irreducible background). The case of fully hadronic decays is further contaminated by pure QCD backgrounds, the more so the smaller the $m_{a_{1}}$ values, so that we have not treated it here. Finally notice the stability of the aforementioned signals against the possibility of stiffer thresholds in the selection of $\tau$ decays, as is made clear by the $P_{T}(\tau)$ dependence of the $S$ and $B$ distributions in the right-hand side of Figs. 7-12 (e.g., an increase of even a factor of two in transverse momentum of the leptonic/hadronic $\tau$ decay products should not dramatically spoil the signal significances in the low $a_{1}$ mass region).

Overall, the $\tau^{+} \tau^{-}$signal yield in the low $a_{1}$ mass region is of order 3000 (for $m_{a_{1}}$ reaching $80 \mathrm{GeV}$ or so) to 30000 (for $m_{a_{1}}$ starting at $10 \mathrm{GeV}$ or so) signal events over a much smaller background, even assuming $\tau^{+} \tau^{-}$resolutions of $10 \mathrm{GeV}$ or so (notice that the width of each histogram in the figures is of $1 \mathrm{GeV}$ and the plots are in log scale).

\section{Conclusions}

In short, we have proven that there exist some regions of the NMSSM parameter space where very light $\mathrm{CP}$-odd Higgs states, with a mixed singlet and doublet nature, could potentially be detected if $m_{a_{1}} \leq M_{Z}$ in the $a_{1} \rightarrow \tau^{+} \tau^{-}$mode if the CP-odd Higgs state is produced in association with a $b \bar{b}$ pair. After a realistic $S / B$ analysis at parton level, we have in fact produced results showing that the extraction of light mass $a_{1} \rightarrow \tau^{+} \tau$ resonances above both the irreducible and (dominant) reducible backgrounds should be feasible using standard reconstruction techniques [24, 25], at least in the double- and single-leptonic decay channels of the $\tau$ 's. While more refined analyses, incorporating $\tau$-decay, parton shower, hadronisation and detector effects, are needed in order to delineate the true discovery potential of the LHC over the actual NMSSM parameter space, we believe that our results are a step in the right direction to prove the existence of a 'More-to-gain theorem' at the CERN collider for the NMSSM with respect to the MSSM, as $\tau^{+} \tau^{-}$signals from such light Higgs bosons are not possible in the latter scenario. Finally, some of the parameter regions where the aforementioned signal can be detected overlap with those where $h_{1,2} \rightarrow a_{1} a_{1}$ decays could also be effective in extracting an $a_{1}$ signal, so that the process discussed here also offers an alternative handle to establish a 'No-lose theorem' for the NMSSM at the LHC.

Further notice that we have explored here the two regimes $2 m_{\tau}<m_{a_{1}}<2 m_{b}$ and $2 m_{b}<m_{a_{1}}$. The former is where the $a_{1} \rightarrow \tau^{+} \tau^{-}$decay rate dominates (these are the points in the 'red island' to the outermost right-hand side of the middle-right plot of Fig. 3), for which $\operatorname{BR}\left(a_{1} \rightarrow \tau^{+} \tau^{-}\right) \geq 0.9$, as the $b \bar{b}$ decay channel is closed. (Our mass point $m_{a_{1}}=9.8 \mathrm{GeV}$ was representative of this situation). The latter is where the $a_{1} \rightarrow b \bar{b}$ decay rate dominates (the corresponding points are all the remaining ones of Fig. 3), since here $\operatorname{BR}\left(a_{1} \rightarrow \tau^{+} \tau^{-}\right) \leq 0.1$ because the $b \bar{b}$ decay channel is open. (Our mass points $m_{a_{1}}=20$, $31,46,60.5$ and $81 \mathrm{GeV}$ were representative of this situation.) 
Unfortunately, a similar analysis in the $a_{1} \rightarrow \gamma \gamma$ channel has showed that the LHC discovery potential is hindered by an overwhelming irreducible background, despite the fact that the $\operatorname{BR}\left(a_{1} \rightarrow \gamma \gamma\right)$ can be equal to unity in some regions of the NMSSM parameter space, a peculiarity of the NMSSM with respect to the MSSM.

\section{Acknowledgments}

We acknowledge useful email exchanges with Cyril Hugonie and Ulrich Ellwanger. We are also very grateful to Lorenzo Basso for technical assistance with some of the plots and to Alexander Belyaev for his help with CalcHEP. This work is supported in part by the NExT Institute. M. M. A. acknowledges a scholarship granted to him by Taibah University (Saudi Arabia).

\section{References}

[1] For reviews, see: e.g., U. Ellwanger, C. Hugonie and A. M. Teixeira, Phys. Rept. 496 (2010) 1 (and references therein); M. Maniatis, Int. J. Mod. Phys. A 25 (2010) 3505 (and references therein).

[2] D.J. Miller, R. Nevzorov and P.M. Zerwas, Nucl. Phys. B 681 (2004) 3.

[3] M. Masip, R. Muñoz-Tapia and A. Pomarol, Phys. Rev. D 57 (1998) 5340.

[4] U. Ellwanger and C. Hugonie, Eur. Phys. J. C 25 (2002) 297.

[5] R. Dermisek and J. F. Gunion, Phys. Rev. Lett. 95 (2005) 041801.

[6] U. Ellwanger, J. F. Gunion and C. Hugonie, JHEP 0507 (2005) 041; U. Ellwanger, J.F. Gunion, C. Hugonie and S. Moretti, hep-ph/0305109 and hep-ph/0401228.

[7] A. Djouadi et al., JHEP 0807 (2008) 002.

[8] J. Dai, J.F. Gunion, R. Vega, Phys. Lett. B 315 (1993) 355 and Phys. Lett. B 345 (1995) 29; J.R. Espinosa, J.F. Gunion, Phys. Rev. Lett. 82 (1999) 1084.

[9] U. Ellwanger, J.F. Gunion and C. Hugonie, hep-ph/0111179, D.J. Miller and S. Moretti, hep-ph/0403137; C. Hugonie and S. Moretti, hep-ph/0110241; A. Belyaev, S. Hesselbach, S. Lehti, S. Moretti, A. Nikitenko and C. H. Shepherd-Themistocleous, arXiv:0805.3505 [hep-ph]; J. R. Forshaw, J. F. Gunion, L. Hodgkinson, A. Papaefstathiou and A. D. Pilkington, JHEP 0804 (2008) 090; A. Belyaev, J. Pivarski, A. Safonov, S. Senkin and A. Tatarinov, Phys. Rev. D 81 (2010) 075021.

[10] S. Moretti and S. Munir, Eur. Phys. J. C 47 (2006) 791.

[11] E. Accomando et al., arXiv:hep-ph/0608079. 
[12] S. Moretti, S. Munir and P. Poulose, Phys. Lett. B 644 (2007) 241.

[13] S. Munir, talk given at the 'International School of Subnuclear Physics, 43rd Course', Erice, Italy, August 29 - Sept. 7, 2005, to be published in the proceedings, preprint SHEP-05-37, October 2005.

[14] D. Zerwas and S. Baffioni, private communication; S. Baffioni, talk presented at "GdR Supersymétrie 2004, 5-7 July 2004, Clermont-Ferrand, France.

[15] U. Ellwanger, J.F. Gunion and C. Hugonie, JHEP 0502 (2005) 066; U. Ellwanger and C. Hugonie, Comput. Phys. Commun. 175 (2006) 290.

[16] See http://www.th.u-psud.fr/NMHDECAY/nmssmtools.html.

[17] S. Schael et al., Eur. Phys. J. C 47 (2006) 547.

[18] R. Dermisek and J. F. Gunion, Phys. Rev. D 76 (2007) 095006.

[19] A. Pukhov, arXiv:hep-ph/0412191.

[20] See http://hep.pa.msu.edu/cteq/public/cteq6.html.

[21] M. M. Almarashi, private programs.

[22] S. Andreas, O. Lebedev, S. R. Sanchez and A. Ringwald, JHEP 1008 (2010) 003.

[23] See, e.g.: F. Sarri, preprint ATL-PHYS-PROC-2008-076 (and references therein); S. Horvat, preprint ATL-PHYS-PROC-2009-063 (and references therein).

[24] ATLAS Collaboration, arXiv:0901.0512 [hep-ex].

[25] CMS Collaboration, J. Phys. G 34 (2007) 995. 

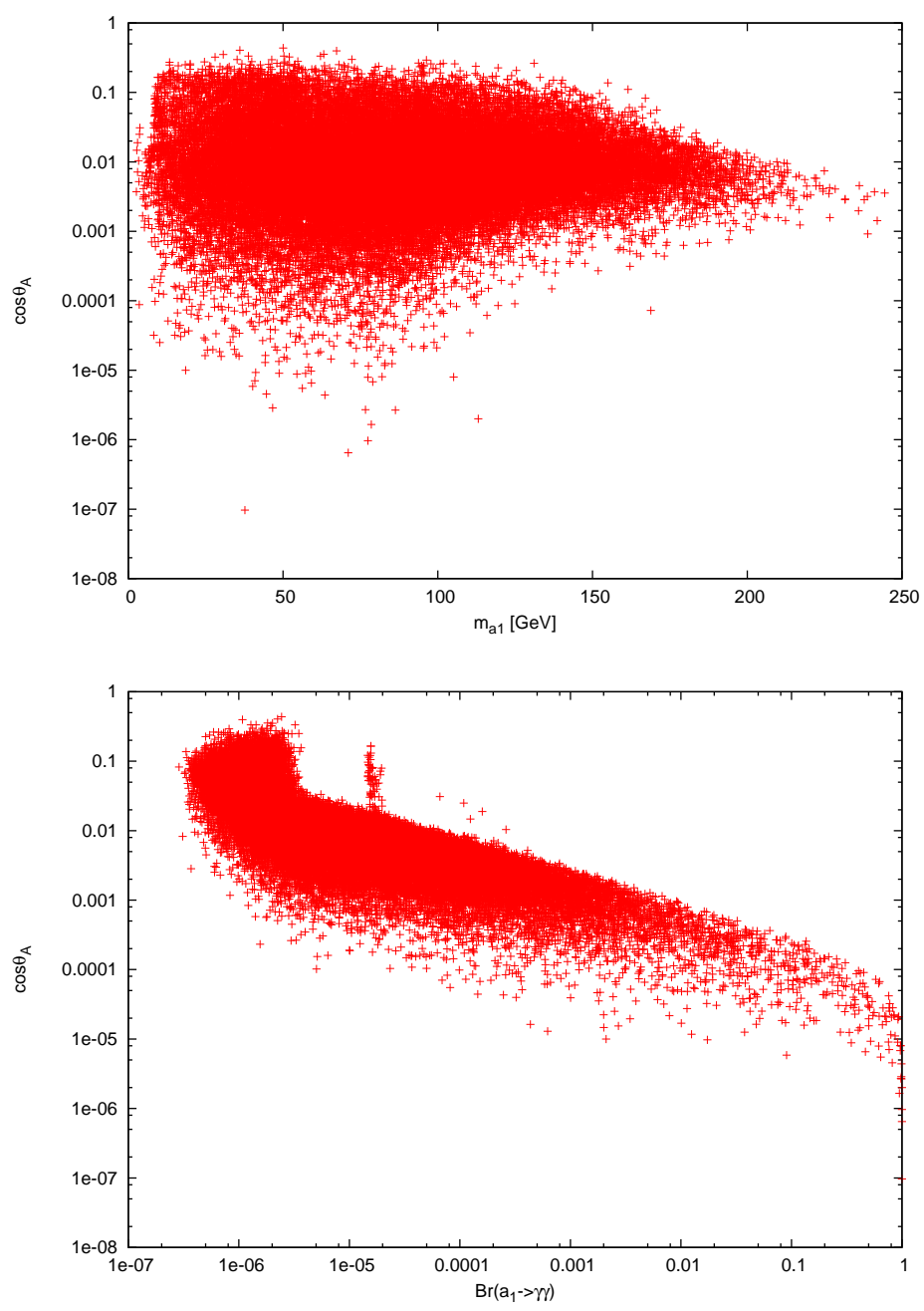

Figure 1: The lightest CP-odd Higgs mass $m_{a_{1}}$ and the $\mathrm{BR}\left(a_{1} \rightarrow \gamma \gamma\right)$ plotted against the mixing angle in the $\mathrm{CP}$-odd sector, $\cos \theta_{A}$. 

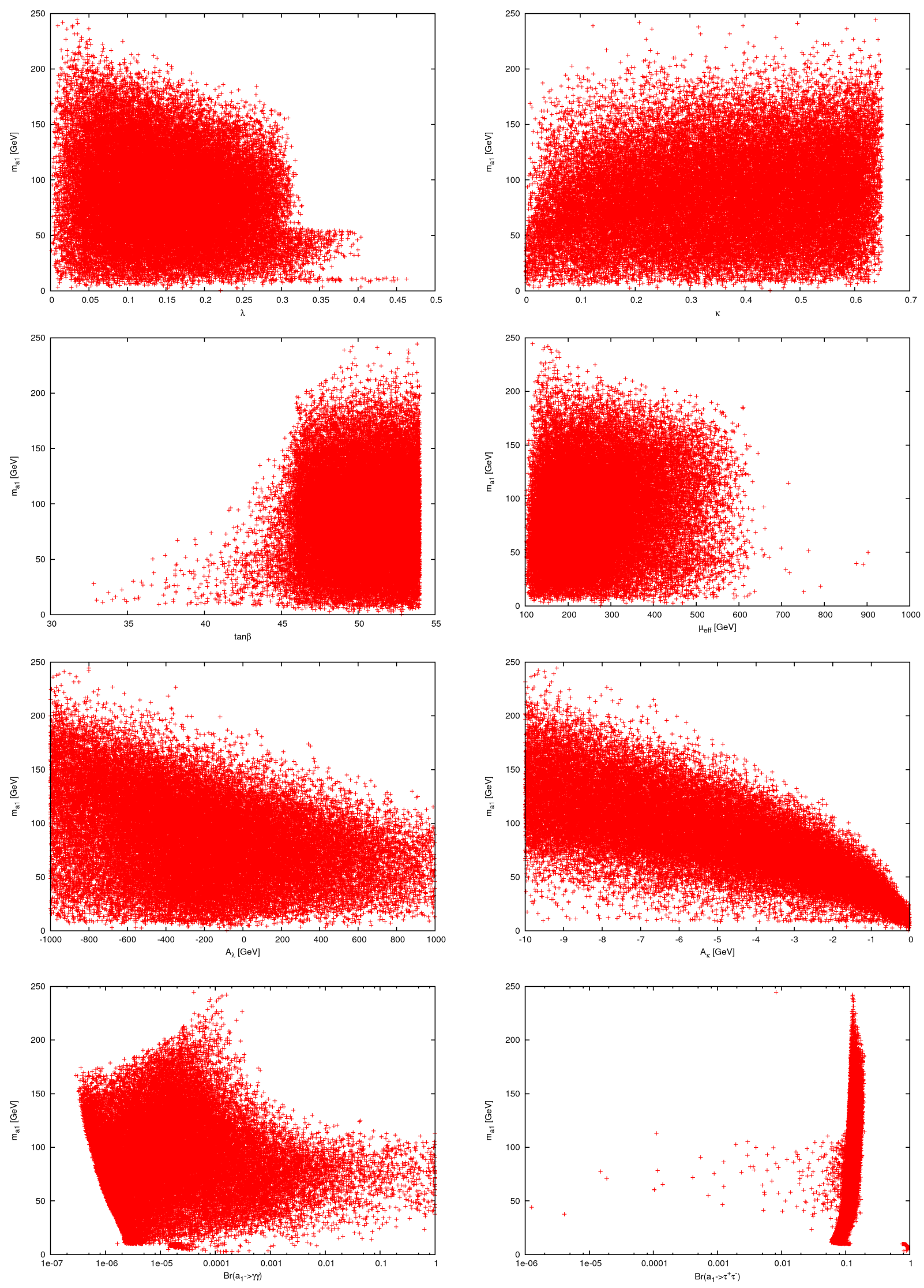

13

Figure 2: The CP-odd Higgs mass $m_{a_{1}}$ as a function of $\lambda, \kappa, \tan \beta, \mu_{\text {eff }}, A_{\lambda}, A_{\kappa}, \operatorname{BR}\left(a_{1} \rightarrow \gamma \gamma\right)$ and $\operatorname{BR}\left(a_{1} \rightarrow \tau^{+} \tau^{-}\right)$. 

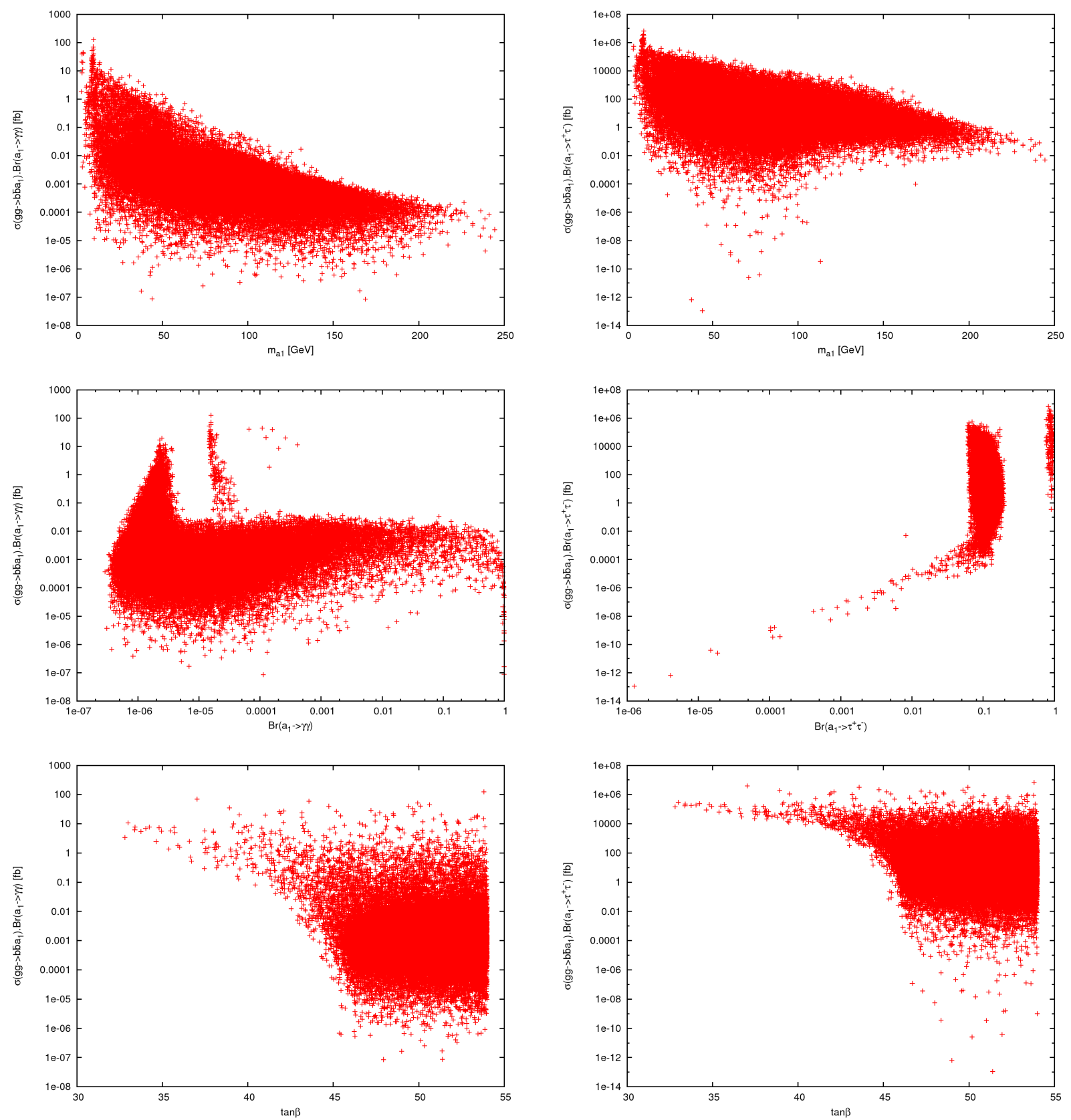

Figure 3: The rates for $\sigma\left(g g \rightarrow b \bar{b} a_{1}\right) \operatorname{BR}\left(a_{1} \rightarrow \gamma \gamma\right)$ (left) and $\sigma\left(g g \rightarrow b \bar{b} a_{1}\right) \operatorname{BR}\left(a_{1} \rightarrow \tau^{+} \tau^{-}\right)$ (right) as functions of $m_{a_{1}}$, BR of the corresponding channel and $\tan \beta$. 

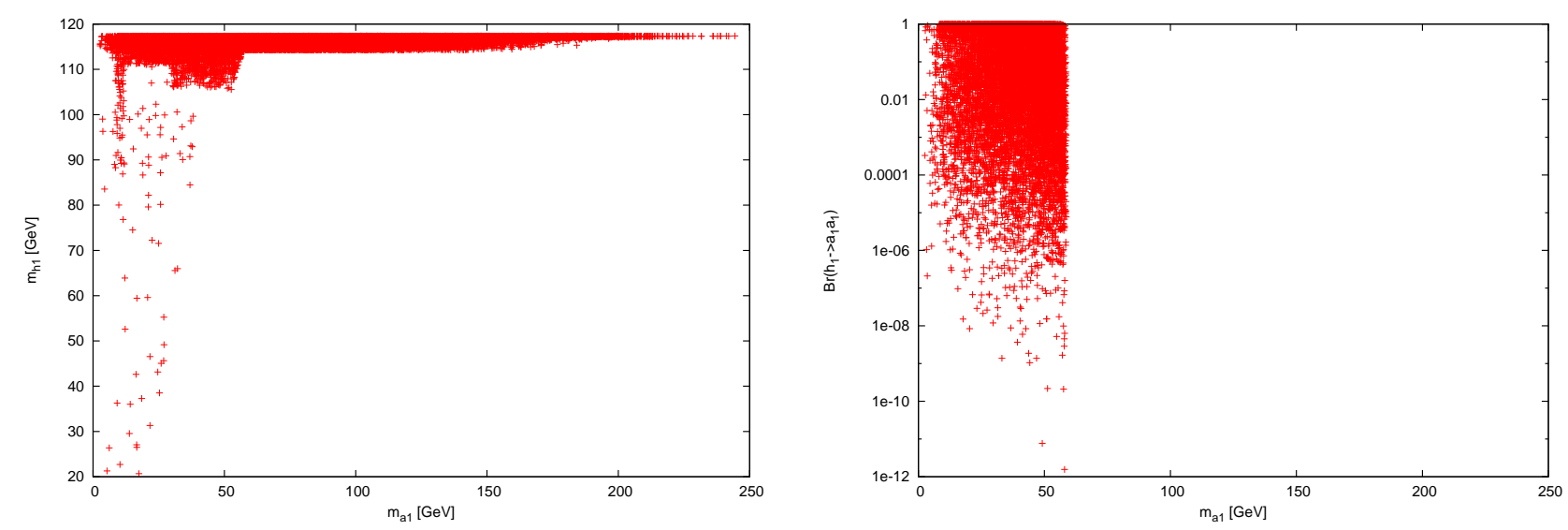

Figure 4: The lightest CP-odd Higgs mass $m_{a_{1}}$ plotted against the lightest CP-even Higgs mass $m_{h_{1}}$ and $\operatorname{BR}\left(h_{1} \rightarrow a_{1} a_{1}\right)$.
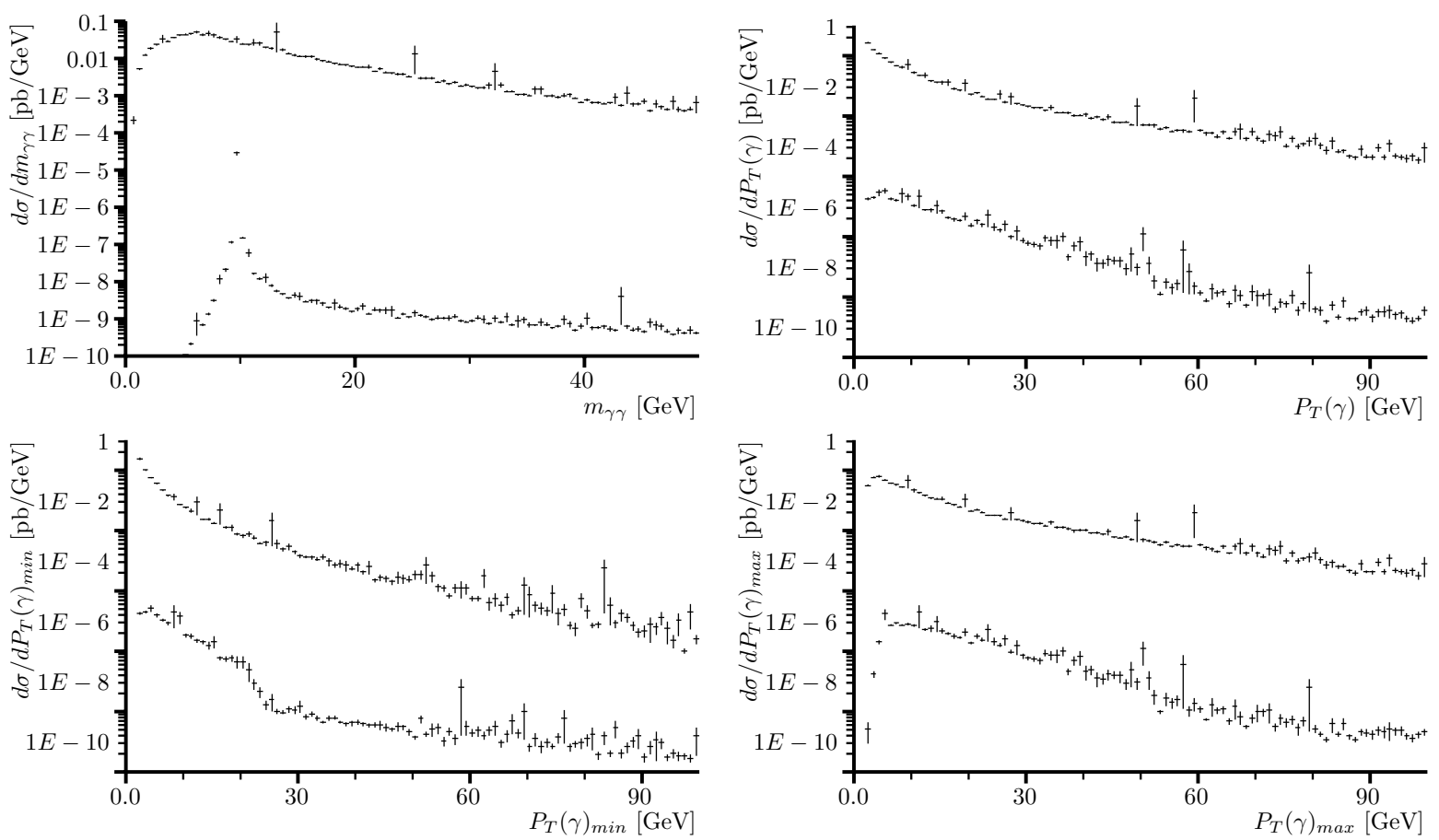

Figure 5: The differential cross section for $m_{a_{1}}=9.8 \mathrm{GeV}$ as a function of the invariant mass $m_{\gamma \gamma}, P_{T}(\gamma), P_{T}(\gamma)^{\min }$ and $P_{T}(\gamma)^{\max }$ for the signal (bottom distribution) only and for the signal and the background together (top distribution), after the cuts in (6). 

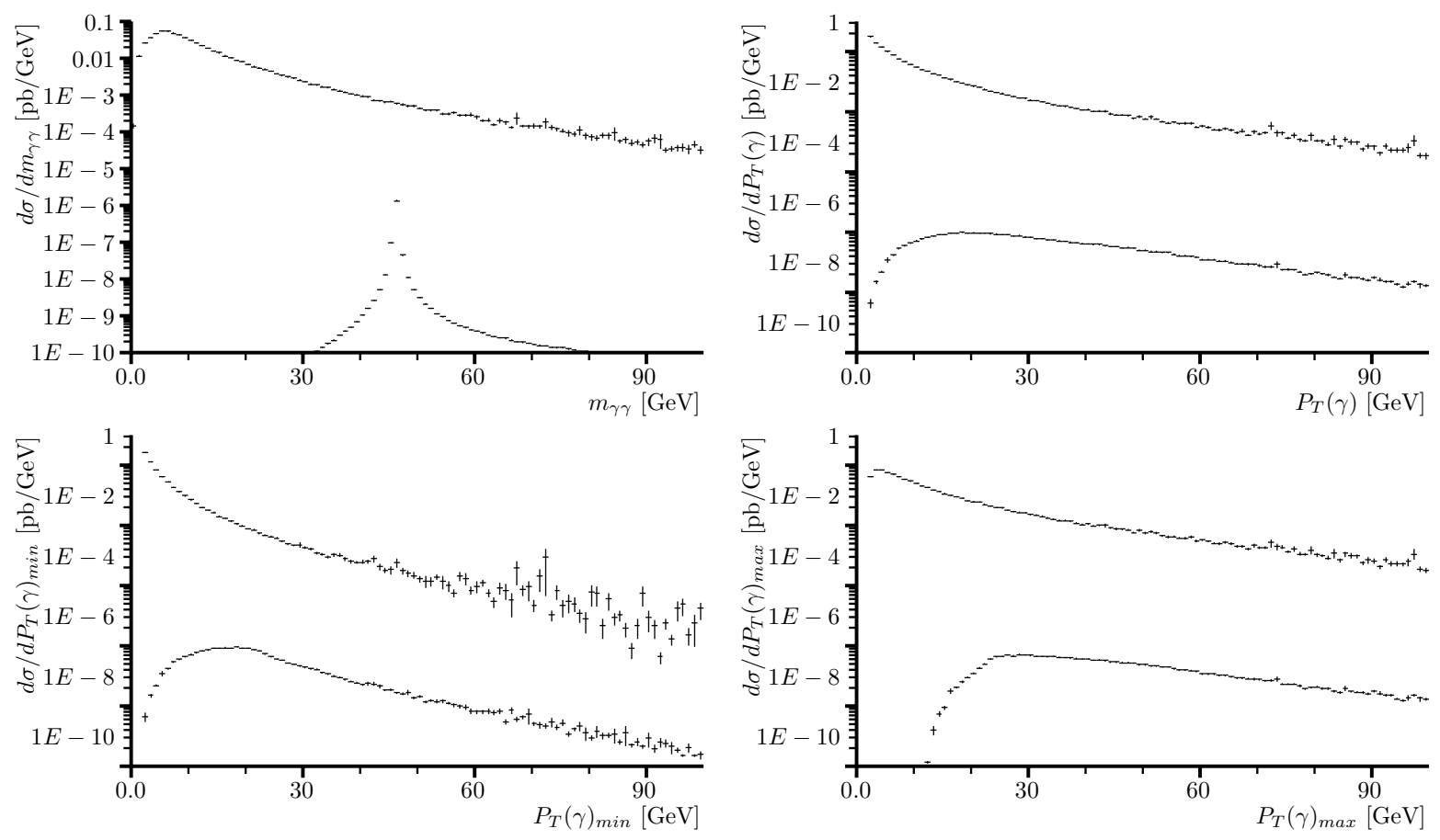

Figure 6: The differential cross section in the $\gamma \gamma$ channel for $m_{a_{1}}=46 \mathrm{GeV}$ as a function of the invariant mass $m_{\gamma \gamma}, P_{T}(\gamma), P_{T}(\gamma)^{\min }$ and $P_{T}(\gamma)^{\max }$ for the signal (bottom distribution) only and for the signal and the background together (top distribution), after the cuts in (6).
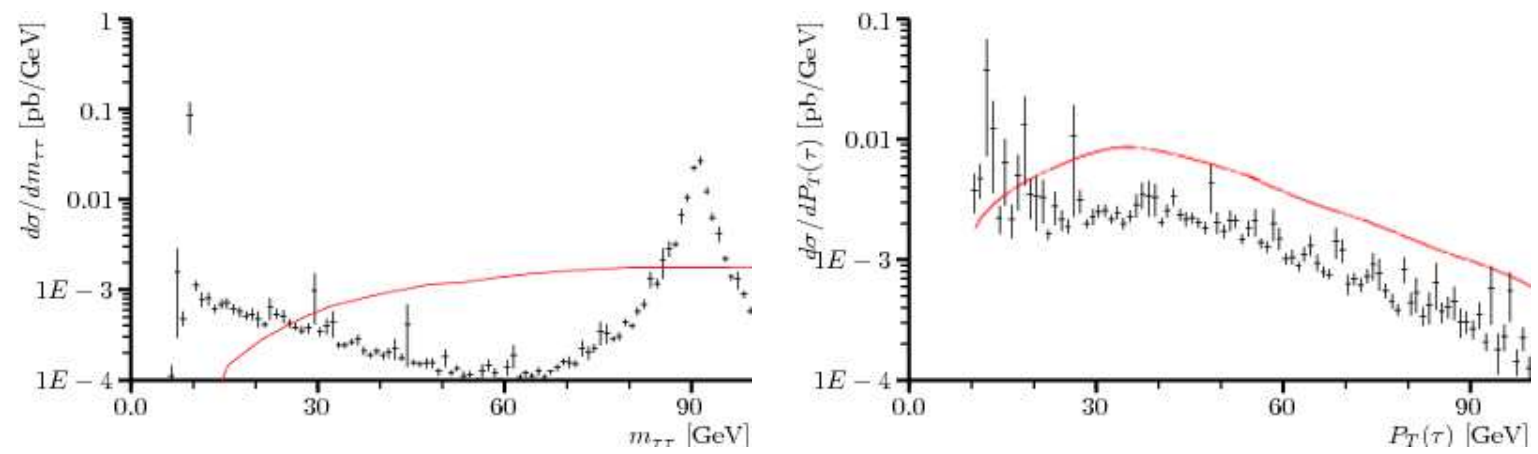

Figure 7: The differential cross section in the $\tau^{+} \tau^{-}$channel for $m_{a_{1}}=9.8 \mathrm{GeV}$ as a function of $m_{\tau \tau}$ (left) and $P_{T}(\tau)$ (right), after the cuts in (7). The histogram points represent the signal and irreducible background together whereas the red line is $t \bar{t}$ background. 

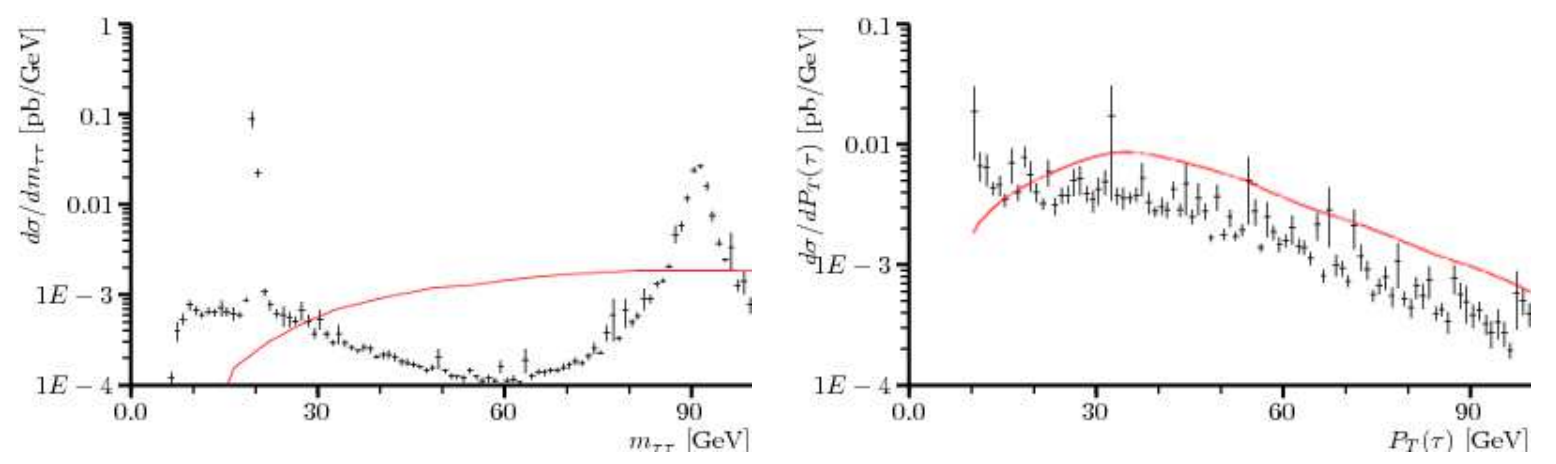

Figure 8: The differential cross section in the $\tau^{+} \tau^{-}$channel for $m_{a_{1}}=20 \mathrm{GeV}$ as a function of $m_{\tau \tau}$ (left) and $P_{T}(\tau)$ (right), after the cuts in (7). The histogram points represent the signal and irreducible background together whereas the red line is $t \bar{t}$ background.
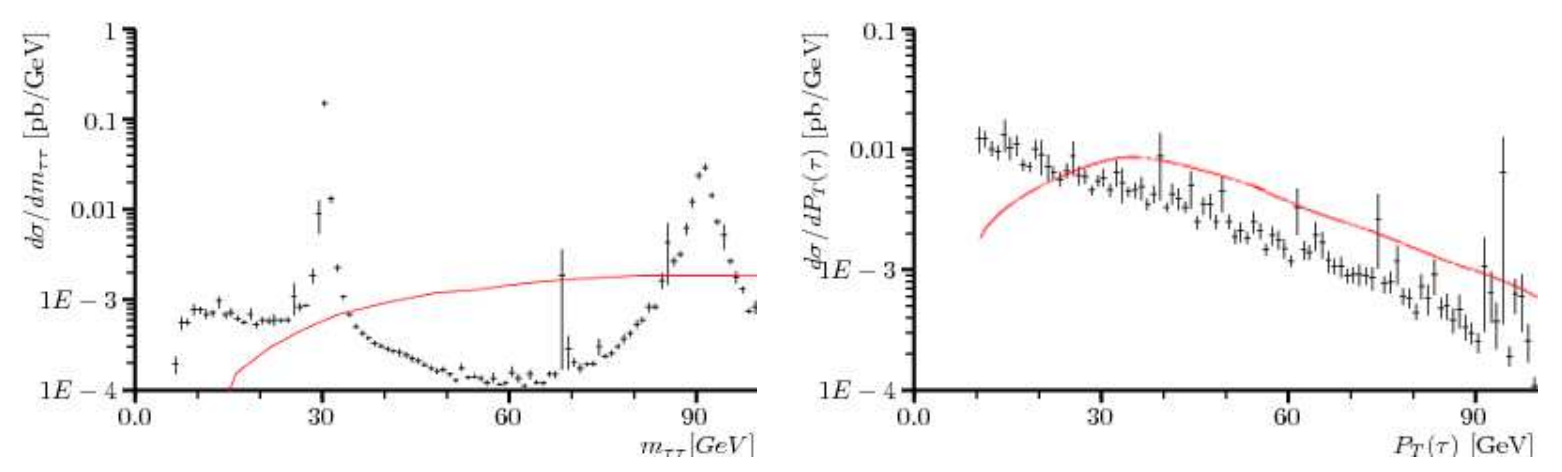

Figure 9: The differential cross section in the $\tau^{+} \tau^{-}$channel for $m_{a_{1}}=31 \mathrm{GeV}$ as a function of $m_{\tau \tau}$ (left) and $P_{T}(\tau)$ (right), after the cuts in (17). The histogram points represent the signal and irreducible background together whereas the red line is $t \bar{t}$ background.
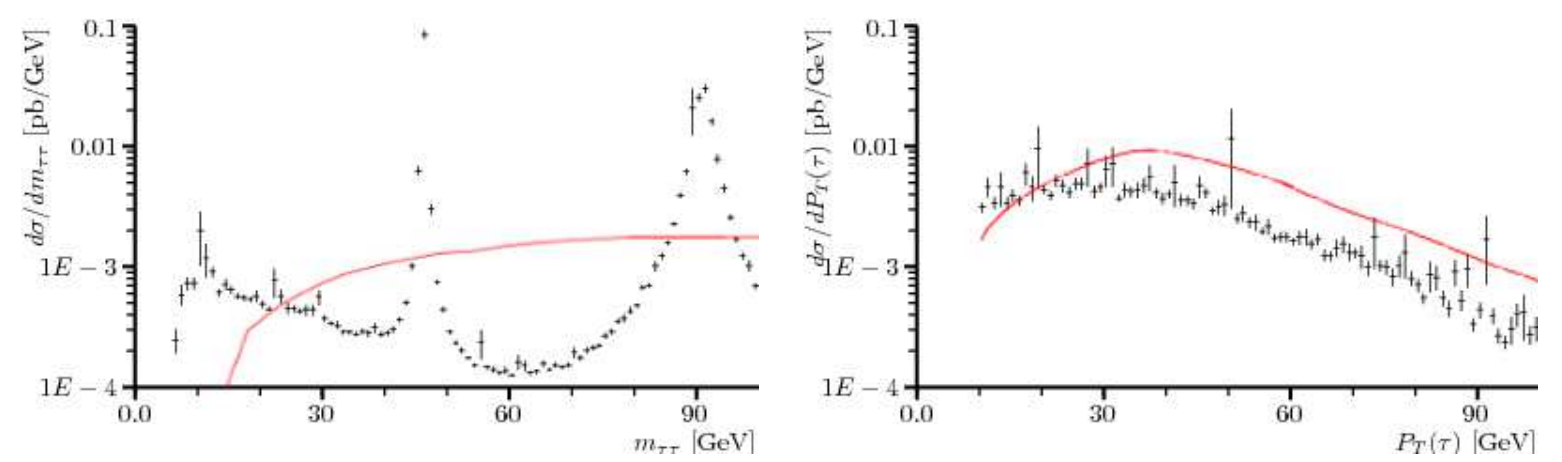

Figure 10: The differential cross section in the $\tau^{+} \tau^{-}$channel for $m_{a_{1}}=46 \mathrm{GeV}$ as a function of $m_{\tau \tau}$ (left) and $P_{T}(\tau)$ (right), after the cuts in (17). The histogram points represent the signal and irreducible background together whereas the red line is $t \bar{t}$ background. 

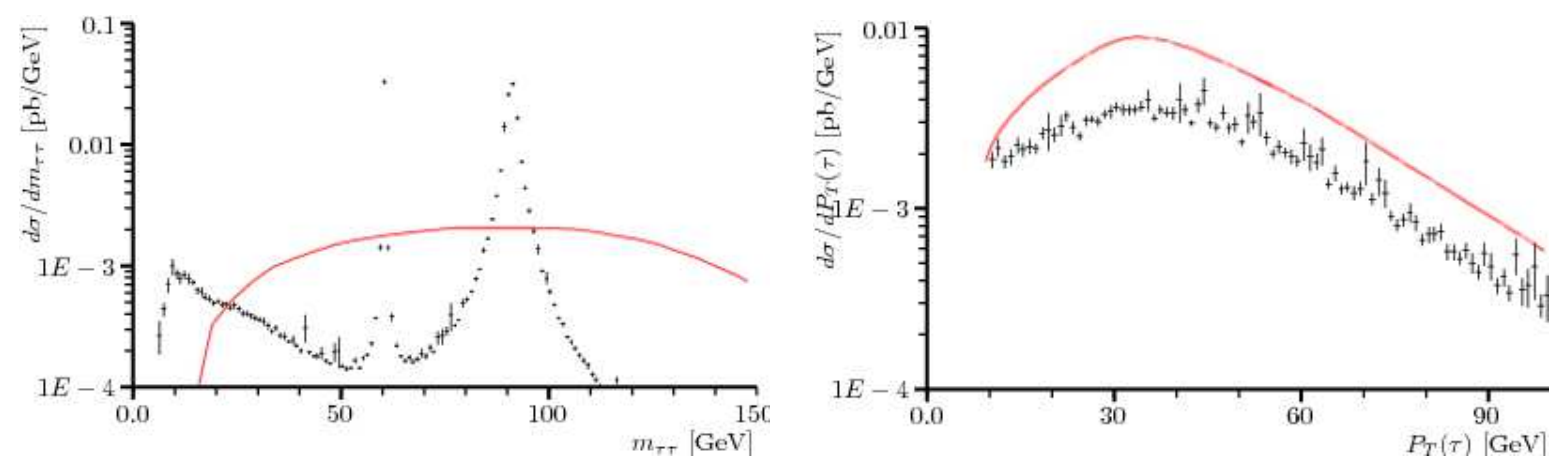

Figure 11: The differential cross section in the $\tau^{+} \tau^{-}$channel for $m_{a_{1}}=60.5 \mathrm{GeV}$ as a function of $m_{\tau \tau}$ (left) and $P_{T}(\tau)$ (right), after the cuts in (7). The histogram points represent the signal and irreducible background together whereas the red line is $t \bar{t}$ background.
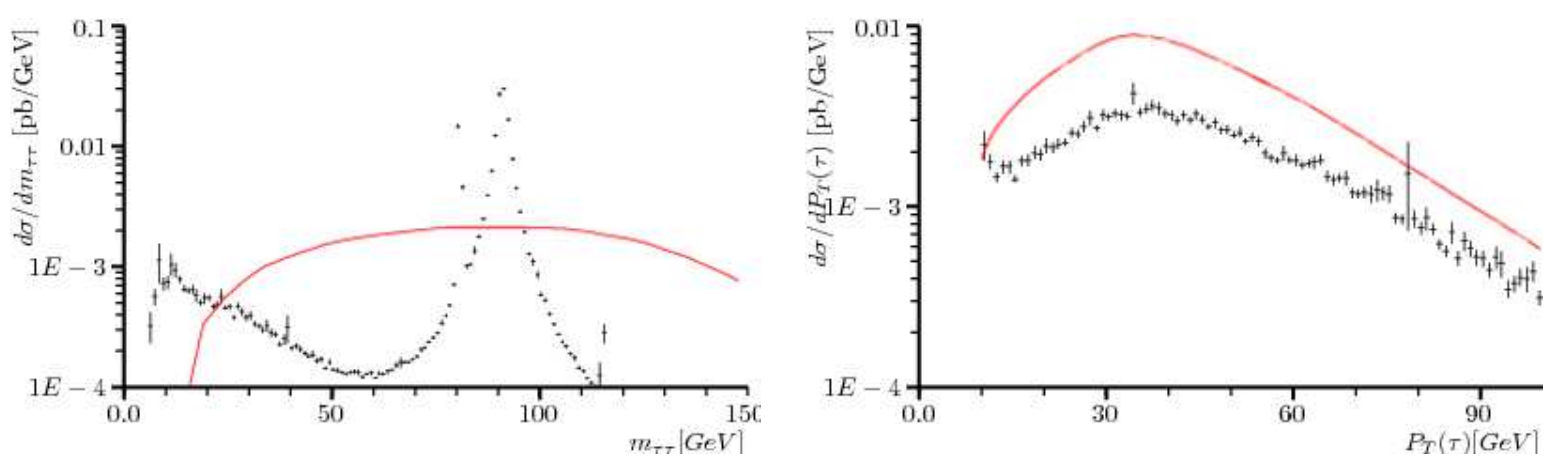

Figure 12: The differential cross section in the $\tau^{+} \tau^{-}$channel for $m_{a_{1}}=81 \mathrm{GeV}$ as a function of $m_{\tau \tau}$ (left) and $P_{T}(\tau)$ (right), after the cuts in (7). The histogram points represent the signal and irreducible background together whereas the red line is $t \bar{t}$ background. 